

\title{
Diferencial de capital humano induzem desigualdades de produtividade do trabalho entre os estados brasileiros
}

\author{
Human capital differentials induce \\ inequalities in labor productivity differentials \\ between Brozilion states
}

Vanecilda de Sousa Borbosa*
José de Jesus Sousa Lemos**

Resumo

Investigou-se o papel do capital humano sobre a produtividade do trabalho nos estados brasileiros entre 2004 e 2015. Capital humano foi medido por duas variáveis: Escolaridade e saúde preventiva. Estimou-se a sinergia de variações de capital humano e produtividade, bem como a relação os impactos das diferenças de capital humano sobre produtividade do trabalho entre os estados. Os resultados mostram que os estados do Sudeste/Sul, mais ricos tem produtividades e capital humano maiores do que os do Nordeste/Norte, mais pobres. Mostraram que Capital humano e produtividade covariam e que diferenciais de capital humano induzem diferenciais de produtividade entre os estados.

Palavras Chave: Desenvolvimento regional; crescimento econômico desigual; capital humano diferenciado.

\begin{abstract}
The role of human capital over labor productivity in the Brazilian States between 2004 and 2005 was investigated. Human capital was measured by two variables: schooling and preventive health. The synergy of variations in human capital and productivity was estimated, as well as the relationship of the impacts of human capital differences ob labor productivity between States. The results show that the richer Southeast/South States have higher productivity and human capital than the poorer Northeast/North. They showed that human capital and productivity would covert and that human capital differentials induce productivity differentials between States.
\end{abstract}

Keywords: Regional Development; Uneven economic growth; Differentiated human capital.

\footnotetext{
* Professora Universidade Federal Rural de Pernambuco UFRPE/UAST. Recife, Pernambuco. https://orcid.org/0000-0003-2117-3421.E-mail: vanysousab@hotmail.com

* Professor Titular, Coordenador do Laboratório do Semiárido Vinculado aos cursos de Mestrado e Doutorado em Economia Rural na Universidade Federal do Ceará. Departamento de Economia Agricola, Centro de Ciencias Agrárias. Campus do PICl. Fortaleza, Ceará. https://orcid.org/0000-0003-1460-0325. E-mail: lemos@ufc.br
} 


\section{INTRODUÇÃO}

Pesquisas de Mincer (1958), Schultz (1962) e Becker (1964), constataram que o Produto Interno Bruto (PIB) de um país é gerado pela junção de dois fatores que incrementam a produtividade do trabalho: capital físico e capital humano. Esses autores criticavam a abordagem clássica que incorporava somente o capital físico para explicar o crescimento.

Ali estavam colocados os alicerces para uma nova maneira de buscar as formas como provavelmente se baseia o crescimento dos países: A teoria do Capital Humano. Essa teoria prescreve que os acessos à educação e à saúde são condicionantes para o aumento da renda Shultz (1962) e Becker (1964). In the theory of human capital, Becker (1964) também mostrou que incrementos em educação provocam incrementos das habilidades e competências dos indivíduos e as suas produtividades.

Por essa razão pessoas que experimentam da privação desses ativos têm dificuldade de acessarem melhores oportunidades de trabalho, com melhores remunerações, tendo em vistas que as atividades que melhor remuneram a força de trabalho, no geral, requerem mão de obra mais qualificada.

A produtividadedotrabalhoéumimportantefatorna determinação do crescimento econômico e o seu diferencial entre Países, Regiões, Estados, Municípios pode ser um dos responsáveis por padrões desiguais de apropriação da riqueza gerada. Nesse sentido a produtividade total dos fatores (PTF), que inclui o capital humano, exerce importante papel na análise de crescimento econômico, pois associa o crescimento da economia às melhorias no processo produtivo, seja por avanços tecnológicos, seja por melhorias nos insumos na gestão da produção, seja pelo aprimoramento da educação e do vigor da força de trabalho (MOREIRA, 1991; MENEZES, 2014).

A dinâmica do setor produtivo é bastante complexa. Vieira Filho e Silveira (2012) ressaltam o papel dos investimentos em processos produtivos, permitindo gerar inovações tecnológicas, combinação eficiente de insumos, que garanta uma maior eficiência no processo produtivo, gerando vantagens comparativas e competitivas. Mas isso apenas é possível se houver uma força de trabalho qualificada. (SHULTZ, 1962; BECKER,1964).

Parte da literatura recente mostra a relação entre capital humano e desenvolvimento econômico. Estudos como os de Johansen (2014); Larianova; Varlamova (2015); Lee; Lee (2018); demonstram que, uma distribuição mais igualitária da educação contribui significativamente para a redução das desigualdades de renda. Há estudos que mostram que a redução das desigualdades na distribuição de renda pode ter sido impactada pelo incremento das habilidades do capital humano e, reciprocamente, melhora na distribuição de renda afetam a qualidade do capital humano. Esta proposição já havia sido defendida por Galor (2012), que mostrou que tanto a teoria como evidencias empíricas demonstraram que a distribuição de renda tem significante impacto sobre a formação do capital humano e sobre a dinâmica do desenvolvimento econômico. 
Assim, se depreende da importância do fator capital humano como fundamental impulsionador da produtividade do trabalho, tendo em vistas que repercute na maior eficiência produtiva, no desenvolvimento e no melhoramento de técnicas que permitem alavancar a produtividade do trabalho e, em consequencia, incrementar a remuneração desse fator, como ensina a teoria neoclássica. (VARIAN, 2015).

Alguns trabalhos, desenvolvidos em diferentes períodos, mostraram essa relação entre qualidade do capital humano, produtividade do trabalho e desenvolvimento. Dentre esses estudos se destacam os de Lucas (1988); Grossman e Helpman (1991); Aghion e Howitt (1992); França, Gasparini e Loureiro (2005); Gray, Jackson e Zhao (2011); Felema et al (2013); Andersen (2015). Todos esses estudos destacam que a capacitação, o vigor da força de trabalho, e os investimentos em pesquisa e desenvolvimento são fatores relevantes que provocam melhores níveis de conhecimento, que viabilizam o domínio de novas tecnologias. Essas permitem alterar as práticas de produção, conduzindo para maiores níveis de produtividades do trabalho e, em decorrência, melhoras na remuneração dos trabalhadores mais qualificados.

É fato que diferenciais na qualidade do capital humano podem induzir a diferenciais em padrões de apropriação de renda, riqueza e promover desigualdades. Diferenciais na qualidade do capital humano induzem crescimento desigual das economias. Além disso, é bastante provável que heterogeneidades observadas ao longo dos anos nas produtividades do trabalho, nos indicadores de capital humano, na formatação da renda e da riqueza de uma localidade acontecem de forma simultânea como se constituíssem num sistema em vasos comunicantes. Variações em torno das médias das produtividades do trabalho nos Estados afetam, e são afetados, sinergicamente, pelas variações em torno das médias dos indicadores de capital humano dos Estados. (ANDERSEN, 2015).

O Brasil é sabidamente um País desigual no que concerne aos padrões de desenvolvimento das suas cinco regiões, dos seus 26 Estados e Distrito Federal. Essas desigualdades nos padrões de desenvolvimento entre as regiões e Estados, bem como dentro das regiões e dos Estados, podem estar associadas às desigualdades na qualidade dos respectivos capitais humanos.

Diante do exposto, este estudo se justifica pela oportunidade de explorar e quantificar as diferenças de dotação de capital humano prevalecentes entre os Estados e o Distrito Federal brasileiros. Capital humano diferenciado provê produtividade do trabalho diferenciada que se reflete nos ganhos também diferenciados dos trabaIhadores, provocando desigualdades na apropriação e na distribuição da riqueza e da renda geradas num sistema econômico. A hipótese central da pesquisa é que os diferenciais de capital humano são os geradores nos padrões de produtividades desiguais entre os Estados brasileiros, sobretudo os situados nas regiões mais desenvolvidas (Sudeste e Sul) em relação àqueles situados nas regiões menos desenvolvidas do País (Nordeste e Norte). 
Assim, os objetivos desta pesquisa são: a - criar o índice de saneamento (ISAN) que é usado no estudo como indicador de saúde preventiva que é um dos itens que compõem o capital humano definido na pesquisa; b - mostrar as desigualdades observadas nas médias das produtividades do trabalho e de indicadores de capital humano entre os Estados e Distrito Federal brasileiros no período de 2004 e 2015; c - mostrar as heterogeneidades existentes entre produtividade do trabalho e capital humano no Distrito Federal e Estados brasileiros no período investigado; d - estimar a relação de causa-efeito das diferenças na dotação de capital humano sobre as diferenças de produtividade do trabalho entre os Estados e Distrito Federal brasileiros.

A partir deste momento do texto, visando evitar repetições que podem se tornar enfadonhas, Estados brasileiros e Distrito Federal (DF) serão tratados indistintamente por Estados, a não ser quando for necessário enfatizar alguma característica ou resultados específicos para o DF. Da mesma forma, produtividade do trabalho será tratada apenas como produtividade.

\section{METODOLOGIA}

Nesta Seção são apresentadas as fontes secundárias dos dados utilizados no estudo, a definição das variáveis utilizadas, as suas aferições. Apresentam-se em seguida os procedimentos metodológicos utilizados para atingir aos objetivos da pesquisa.

Definições e justificativas dos indicadores e fontes dos dados utilizados na pesquisa.

O indicador de produtividade é aferido pela relação entre os PIB agregados dos Estados e a população economicamente ativa (PEA), no período compreendido entre os anos de 2004 e 2015. Até 2010 o IBGE definia como PEA a população entre 10 e 65 anos. A partir de 2011 a idade mínima da PEA ascendeu para 15 anos. Neste trabalho a produtividade se estima dividindo-se o PIB agregado dos Estados e corrigido para valores de 2015, pela população maior de 15 anos. Calcula-se em seguida o valor médio dessas produtividades para o período estudado, bem como os respectivos coeficientes de variação (CV).

Os indicadores de capital humano utilizados no estudo são: escolaridade e saúde preventiva. A métrica do indicador de escolaridade é o percentual da população maior de 15 anos, em cada Estado, que possua mais de nove (9) anos de estudos. Este indicador foi assim dimensionado porque, segundo a Resolução CNE/CEB n 3/2005 de 3/08/2005 do Ministério da Educação (MEC), nove anos é o tempo mínimo para a conclusão do ensino fundamental. Na pesquisa se considera que a qualificação minimamente adequada para a força de trabalho lograr padrões minimamente satisfatórios de produtividade seria ter essa escolaridade. 
A pesquisa afere a saúde de forma preventiva. Para tanto se desenha um modelo de agregação dos ativos ambientais aferidos da seguinte forma: percentual da população que teve acesso à água encanada, saneamento minimamente adequado e coleta sistemática de lixo, direta ou indiretamente. A agregação ponderada desses três ativos é feita no índice de saneamento (ISAN), que é construído no estudo. Esses indicadores são importantes porque proporcionam mais vigor aos seres humanos, tendo em vistas que preparam o organismo para ter maior resistência e um melhor resiliência aos problemas de saúde. (UNITED NATIONS, 2006; UNITED NATIONS, 2014).

$\mathrm{O}$ período de tempo para a coleta dos dados ficou condicionado à sua disponibilidade pelo IBGE. Antes de 2004 as PNAD Anuais não forneciam informações de acesso à água encanada, saneamento e coleta sistemática de lixo para os Estados da região Norte. Por outro lado, a última PNAD Anual foi publicada pelo IBGE em 2015. A partir de 2016 o IBGE publica as PNAD Contínuas. Esses documentos deixaram de registrar indicadores de acesso aos serviços de água encanada, saneamento e coleta sistemática de lixo. Como a pesquisa foi desenhada para avaliar todos os Estados e utilizar esses ativos ambientai como indicadores de saúde preventiva, teve que adotar como período de avaliação os anos de 2004 e 2015. A outra fonte de informação, também retirada do IBGE, é extraída do link "PIB dos municípios".

\section{Construção do ISAN através de anólise fatorial (AF): Primeiro objetivo.}

A pesquisa buscou estimar um único índice de saneamento (ISAN) para ser usado como aferidor de saúde preventiva. Buscou-se um instrumento de aferição que fosse capaz fazer o mapeamento de todos os Estados brasileiros nos anos avaliados na pesquisa. Assim procedendo os ISAN dos Estados serão diretamente comparáveis. A equação de definição do índice de saneamento (ISAN) é a seguinte:

$$
\operatorname{ISAN}_{\mathrm{t}}=\sum \mathrm{w}_{\mathrm{it.}} \mathrm{X}_{\mathrm{it}}:(\mathrm{i}=1,2, \ldots, 27) ;(\mathrm{t}=2004,2005, \ldots, 2015) \text {. }
$$

Na equação (1) $w_{i t}\left(0<w_{i t}<1 ; e \sum w_{i t}=1\right)$ são os pesos associados aos indicadores $X_{i t}$. Os $X_{\text {it }}$ são definidos para o período de 2004 a 2015, da seguinte forma:

$\mathrm{X}_{1 \mathrm{t}}=$ percentagem da população do i-ésimo Estado com acesso à água encanada no t-ésimo ano;

$\mathrm{X}_{2 \mathrm{t}}=$ percentagem da população do i-ésmo Estado que tem acesso à rede de esgotamento sanitário ou à fossa séptica que esteja conectada, ou não, à rede geral, no ano " $\mathrm{t}$ ";

$\mathrm{X}_{3 \mathrm{t}}=$ percentual da população do i-ésimo Estado com acesso à coleta sistemática de lixo, de forma direta ou indireta, no ano "t".

Na construção do ISAN foi necessário estimar os pesos associados às variáveis que o compõem. Para este estudo a estratégia utilizada foi mediante a busca das interrelações que existem entre os seus indicadores. Isso foi buscado usando análise fatorial (AF) ancorada na técnica de decomposição em componentes principais. 
A análise fatorial é procedimento estatístico utilizado para estudar modelos envolvendo mais de duas variáveis aleatórias, inter-relacionadas, de modo que seus diferentes efeitos não possam ser interpretados de forma separada e que não haja uma definição clara de causa e efeito entre elas (FÁVERO et al., 2009).

Para avaliar se o método de análise fatorial se adéqua para a realização de estudos como este, é necessário que a matriz de correlação entre as variáveis não seja uma identidade. Isso implica que essa matriz tenha valores unitários na diagonal principal e nas diagonais secundárias variáveis que são correlacionadas. Além disso, para verificar a possibilidade de aplicar a AF, é necessário aferir a medida de adequação da amostra. Para tanto são realizados dois procedimentos estatísticos: Teste de "esfericidade" de Bartlett e Medida de "adequacidade" da amostra de Kaiser-Meyer-Olkin (KMO).

O teste de "esfericidade" de Bartlett testa a hipótese de que as variáveis não sejam correlacionadas na população. A hipótese básica assume que a matriz de correlação da população é uma identidade e, por isso, a AF é inapropriada. A hipótese alternativa é que a matriz não seja identidade. Para aplicar essa técnica no estudo somente será possível se a hipótese nula for rejeitada em beneficio da hipótese alternativa.

A Medida de "adequacidade" da amostra de Kaiser-Meyer-Olkin é aferida pelo índice (KMO). Os valores desse índice, que indicam se a AF é apropriada, variam de autor para autor. Para Hair et al (1988) são valores aceitáveis para o KMO os que estejam contidos no intervalo entre 0,5 a 1,0. Portanto, abaixo de 0,5 indica que a AF é inadequada.

Além desses testes se estima o percentual de variância explicada pelos componentes estimados. Quanto mais próxima de 100\% for a variância explicada, melhor será o ajustamento obtido. Leitores interessados em buscar mais detalhes acerca dos métodos de análises multivariadas em geral, e fatorial em particular, podem encontrar nos seguintes autores: Dillon, Goldstein (1984); Favero et al (2009); Bezerra (2016); Lira et al (2017).

Como os indicadores que fazem parte do ISAN são construídos em percentuais, este índice, por construção, varia entre zero e cem. Mais próximo de cem, melhor o padrão de saúde aferido indiretamente ao acesso aos ativos ambientais de água encanada, saneamento e coleta sistemática de lixo. Mais próximo de zero, menor é o percentual de acesso da população do Estado a esses ativos de forma devidamente ponderada.

Realizada a etapa de preparação dos dados, procede-se a AF para gerar os componentes e os escores fatoriais. A partir desses componentes e/ou dos escores fatoriais (indistintamente) é que são gerados os pesos utilizados na ponderação de cada um dos indicadores do ISAN, mostrados na Equação (1). 


\section{ESTATÍSTICAS DESCRITIVAS DE PRODUTIVIDADE E CAPITAL HUMANO: SEGUNDO E TERCEIROS OBJETIVOS}

Para alcançar o segundo objetivo da pesquisa estimam-se estatísticos descritivos associados a cada um dos indicadores: Produtividade e de capital humano utilizados na pesquisa.

Para captar as heterogeneidades associadas às variáveis empregadas na pesquisa (terceiro objetivo) utilizam-se como instrumento de aferição os respectivos coeficientes de variação (CV). Aferir as homogeneidades/heterogeneidades associadas a esses indicadores proporcionam informação acerca da forma como eles evoluíram no tempo em torno do valor esperado.

Por definição o CV afere a relação percentual entre o desvio padrão e a média aritmética de uma variável aleatória. O CV tem a utilidade de medir a heterogeneidade ou a homogeneidade que se observa na distribuição dos valores de uma variável aleatória em torno do seu valor esperado. (GOMES, 1985; GARCIA, 1989; SORENSEN, 2000; VAZ et al, 2017).

A vantagem de usar o CV nesse tipo de avaliação, em relação a outras medidas de variabilidade, é que é invariante com as unidades de medidas. Ainda permite a comparação de desigualdades ou de heterogeneidades entre variáveis aferidas em diferentes unidades de medida. (GARCIA, 1989; O'REILLY et al, 1989; WIERSEMA, BANTEL,1993; SORENSEN, 2000; FAO, 2006; FAO, 2014).

Quanto mais próximo de zero for o CV associado à distribuição de uma variável aleatória, mais homogênea, ou mais estável, será a distribuição das observações em torno da média. Embora não tenha limite superior, para usar o CV como indicador de aferição de homogeneidade ou de heterogeneidade da distribuição de uma variável aleatória, pode-se recorrer à definição de seus valores críticos mínimos, para ajudar na sua interpretação. Gomes (1985) estabeleceu limites gerais para classificação dos CV em experimentos agrícolas. Serão esses valores a serem utilizados nesta pesquisa como referencias para as magnitudes dos CV. (Quadro1).

Quadro 1. Classificação do Coeficiente de Variação (CV) de acordo com a sua amplitude.

\begin{tabular}{|l|l|}
\hline Classificação do CV & Amplitude do CV \\
\hline Baixo & $\mathrm{CV}<10 \%$ \\
\hline Médio & $10 \% \leq \mathrm{CV}<20 \%$ \\
\hline Alto & $20 \% \leq \mathrm{CV}<30 \%$ \\
\hline Muito alto & $\mathrm{CV} \geq 30 \%$ \\
\hline
\end{tabular}

Fonte: GOMES, 1985.

Neste estudo os níveis de heterogeneidades associados aos indicadores utilizados também serão assim classificadas: baixos, médios, altos e muito altos quando os respectivos CV alcançarem as magnitudes referidas no Quadro 1. 
Relação entre os diferenciais de produtividade e de capital humano: quarto objetivo.

Para o quarto objetivo a pesquisa projetou aferir os efeitos das diferenças dos indicadores de capital humano entre os Estados sobre as diferenças de produtividade. $O$ procedimento adotado seguiu duas etapas. Na primeira hierarquizam-se, em ordem decrescente, os Estados segundo as respectivas médias de produtividades. Em seguida calculam-se as diferenças entre as maiores médias de produtividade e todas as outras médias menores de produtividade.

Assume-se que as diferenças de produtividades de cada Estado são afetadas pelas respectivas diferenças dos indicadores de escolaridade e de saúde preventiva (ISAN). Ao Estado "i" estão associados a produtividade $\left(\mathrm{P}_{\mathrm{i}}\right)$, a escolaridade $\left(\mathrm{E}_{\mathrm{i}}\right)$ e o ISAN $\left(\mathrm{I}_{\mathrm{i}}\right)$. Ao Estado "j" estão associados a produtividade $\left(\mathrm{P}_{\mathrm{j}}\right)$, a escolaridade $\left(\mathrm{E}_{\mathrm{j}}\right)$ e o ISAN (I).

Definem-se as seguintes diferenças: $\left(P_{i}-P_{j}\right) ;\left(E_{i}-E_{j}\right)$ e $\left(I_{i}-I_{j}\right)$. Por construção: $\left(P_{i}-P_{j}\right)$ será estritamente positivo para todos os "i" e "j". As diferenças $\left(E_{i}-E_{j}\right)$ e $\left(I_{i}-I_{j}\right)$ podem assumir valores nulos, positivos ou negativos. Espera-se que prevaleçam diferenças positivas para suportar a hipótese de que os diferenciais de produtividade são afetados positivamente pelos diferencias dos indicadores de capital humano. Chamando:

$$
\begin{aligned}
& d\left(P_{i j}\right)=\left(P_{i}-P_{j}\right)>0 \text { para todos os Estados "i" } e^{\prime \prime} j " ; \\
& d\left(E_{i j}\right)=\left(E_{i}-E_{j}\right) ; \text { maior, menor ou igual a zero; } \\
& d\left(I_{i j}\right)=\left(I_{i}-I_{j}\right) ; \text { maior, menor ou igual a zero. }
\end{aligned}
$$

Define-se a equação (2) que é utilizada no estudo para estimar os impactos dos diferenciais de capital humano sobre os diferenciais de produtividade:

$$
d\left(P_{i j}\right)=\beta_{0}+\beta_{1}\left[d\left(E_{i j}\right)\right]+\beta_{2}\left[d\left(I_{i j}\right)\right]+\xi_{i j} .
$$

Na equação (2) o coeficiente linear $\beta_{0}$ será o valor médio de $d\left(P_{i j}\right)$ quando, e se, os diferenciais de escolaridade $\left[\mathrm{d}\left(\mathrm{E}_{\mathrm{ij}}\right)\right]$ e de saneamento $\left[\mathrm{d}\left(\mathrm{I}_{\mathrm{ij}}\right)\right]$ forem ambos nulos. Também será nulo se essas variáveis não influenciarem os diferenciais de produtividade. Isso ocorrerá se os coeficientes angulares $\beta_{1}$ e $\beta_{2}$ não forem estatisticamente diferentes de zero. $O$ que se espera é que esses coeficientes angulares, além de estatisticamente diferentes de zero, sejam positivos. Eles aferem a sensibilidade dos diferenciais de produtividade em decorrência das variações nos diferenciais de escolaridade e de saúde preventiva, respectivamente. $O$ ruído aleatório $\xi_{i^{\prime}}$, por hipótese, se distribui com média zero, variância constante e não é autoregressivo. Pressupostos que garantem as estimações dos parâmetros $\beta_{0^{\prime}} \beta_{1}$, e $\beta_{2}$ pelo método dos mínimos quadrados ordinários. (WOOLDRIDGE, 2012).

\section{RESULTADOS}

A apresentação dos resultados inicia com a estimação dos pesos que foram utilizados para a construção do índice de saneamento (ISAN) utilizado como medida 
de saúde preventiva neste estudo. Em seguida os resultados serão apresentados e discutidos na cronologia em que foram apresentados os objetivos.

\section{Resultados para a estimação do ISAN.}

Para utilizar a técnica de decomposição em componentes principais, o primeiro teste feito foi verificar se a matriz de correlação entre os indicadores utilizados na pesquisa para construir o ISAN não é uma identidade (Teste de Bartllet). Isso foi comprovado tendo em vistas que os coeficientes de correlação de Pearson estimados para as três variáveis que o compõem (acesso à água, acesso ao saneamento, acesso à coleta de lixo), além de serem estatisticamente diferentes de zero, são todos superiores a 0,5. O teste de $\mathrm{KMO}=0,696$ indica que a $\mathrm{AF}$ se adéqua à estimação do ISAN com os indicadores propostos. A variância explicada pelo único fator criado é de 69,15\%.

Desta forma foi possível estimar os pesos associados aos indicadores do ISAN. Todas as estatísticas relevantes para a estimação do único fator, bem como os pesos gerados a partir dos componentes e/ou dos escores fatoriais estão mostrados na Tabela 1.

\footnotetext{
Tabela 1: Componentes, escores fatoriais, variâncias explicadas e teste KMO associados à aplicação do método de decomposição em componentes principais para a estimação dos pesos do ISAN nos Estados e no Distrito Federal brasileiros entre 2004 e 2015.
}

\begin{tabular}{|c|c|c|c|}
\hline \multicolumn{4}{|c|}{ Componentes Principais Para as Cinco Regiões (Geral) } \\
\hline Variáveis & Componentes & Escores & Pesos \\
\hline$X_{1 t}$ & 0,850 & 0,410 & 0,34 \\
\hline$X_{2 t}$ & 0,805 & 0,388 & 0,32 \\
\hline$X_{3 t}$ & 0,839 & 0,405 & 0,34 \\
\hline \multicolumn{4}{|c|}{$\begin{array}{l}\text { Variância Explicada pelo Componente Fatorial: } 69,15 \% \text {; } \\
\text { KMO = 0,696. Teste de Bartlett: Matriz não é identidade } \\
\text { tendo em vistas os coeficientes de correlação serem } \\
\text { positivos e diferentes de zero. }\end{array}$} \\
\hline
\end{tabular}

Fonte: Estimativas feitas pelos autores da pesquisa.

Com base nos pesos estimados na pesquisa, apresenta-se a equação do ISAN, tal como estimada para aferir saúde preventiva nesta pesquisa. Observa-se que os pesos associados ao acesso à água encanada $\left(\mathrm{X}_{1 \mathrm{t}}\right)$ e à coleta sistemática de lixo $\left(\mathrm{X}_{3 \mathrm{t}}\right)$ foram iguais $(0,34)$ e que o peso associado ao saneamento $\left(X_{2 t}\right)$ foi ligeiramente menor, $(0,32)$. Esses resultados sinalizam que o ISAN, calculado dessa forma, tem participação praticamente equitativa dos indicadores. Assim, os eventuais sobressaltos no ISAN decorrerão dos valores observados dos indicadores de acesso à água encanada, saneamento, e coleta de lixo em cada Estado, não em decorrência dos pesos estimados que decorrem do método utilizado. A equação do ISAN fica definida como segue:

$$
\operatorname{ISAN}_{t}=0,34 X_{1 t}+0,32 X_{2 t}+0,34 X_{3 t}
$$




\section{DESIGUALDADES ENTRE AS PRODUTIVIDADES DO TRABALHO E CAPITAL HUMANO}

Os resultados encontrados na aferição das estatísticas descritivas que mostram as produtividades do trabalho, escolaridade e ISAN dos Estados e o Distrito Federal estão apresentados na Tabela 2.

A produtividade varia de $\mathrm{R} \$ 7.499,36$ no Piauí a $\mathrm{R} \$ 41.869,33$ no Distrito Federal. Assim, no DF a média da produtividade equivaleu a 5,6 vezes aquela que foi estimada para o Piauí. Também se observa pelas evidencias mostradas na Tabela 2 que, no conjunto, os Estados do Nordeste apresentam as menores médias de produtividades, e os Estados do Sudeste apresentam as maiores médias. Com efeito, a média de produtividade estimada para o Nordeste foi de $\mathrm{R} \$ 11.091,34$, ao passo que a Sudeste foi de $\mathrm{R} \$ 30257,05$. (Tabela 2).

Assim, a produtividade do Nordeste representa apenas $37 \%$ da estimada para o Sudeste no período investigado. Além disso, a produtividade estimada para o Nordeste representa 39\% daquela estimada para o Centro Oeste; 39,5\% da média do Sul; e 75,3\% da média observada para o Norte. Portanto, no Nordeste se praticaram as menores produtividades aferidas pela sua média estimada para o período 2004/2015. (Tabela 2).

Em relação ao comportamento dos Estados, observa-se que na parte superior do ranking da produtividade, logo abaixo do Distrito Federal, seguem os Estados de São Paulo $(\mathrm{R} \$ 38.016,42)$ e Rio de Janeiro $(\mathrm{R} \$ 32.891,58)$. Na parte inferior do ranking, estão os Estados da Paraíba (R\$ 9.148,75) e do Maranhão (R\$ 9.279,17) que, juntos com o Piauí, se constituem os Estados com as menores médias de produtividade entre 2004 e 2015. (Tabela 2).

Em termos de escolaridade, observa-se que nos Estados do Nordeste prevalecem as menores médias das populações maiores de 15 anos que estudaram além do nível fundamental. A média estimada para essa região é de apenas $31,21 \%$ da população maior de 15 anos que estudou além do nível fundamental. No Sudeste (43,66\%) esta é a maior média da população regional maior de 15 anos que estudou mais de nove anos. (Tabela 2).

Nos extremos estão Alagoas em que a média da população maior de 15 anos que estudou além do nível fundamental é de apenas $25,62 \% \%$. No topo desse ranking está o Distrito Federal, para onde se estimou uma média de $56,63 \%$ da população maior de 15 anos que estudou mais de nove anos. São Paulo (49,29\%) e Rio de Janeiro (47,28\%) completam os três Estados da parte superior desse ranking. Piauí (28,0\%) e Maranhão $(29,24 \%)$ completam, com Alagoas, os três Estados com os menores percentuais de população maior de 15 anos que apenas estudou até o nível fundamental. (Tabela 2).

Está também no Sudeste o maior ISAN estimado (maior percentual ponderado de população com acesso à água encanada, saneamento e coleta sistemática de lixo). Para essa região o percentual estimado foi de $89,43 \%$. A região Norte apresenta o menor 
Tabela 2: Média e Coeficiente de Variação (CV) da Produtividade, da Escolaridade e do ISAN entre 1990 e 2015.

\begin{tabular}{|c|c|c|c|c|c|c|}
\hline \multirow[b]{2}{*}{ Estado } & \multicolumn{2}{|c|}{ Produtividade } & \multicolumn{2}{|c|}{ Escolaridade } & \multicolumn{2}{|c|}{ ISAN } \\
\hline & $\begin{array}{c}\text { Média } \\
\text { (R\$) }\end{array}$ & $\begin{array}{l}\text { CV } \\
\text { (\%) }\end{array}$ & $\begin{array}{l}\text { Média } \\
(\%)\end{array}$ & $\begin{array}{l}\mathrm{CV} \\
(\%)\end{array}$ & $\begin{array}{l}\text { Média } \\
(\%)\end{array}$ & $\begin{array}{l}\text { CV } \\
(\%)\end{array}$ \\
\hline \multicolumn{7}{|l|}{ Norte } \\
\hline $\mathrm{AC}$ & 12559,59 & 15,44 & 35,17 & 14,71 & 61,10 & 7,73 \\
\hline AM & 22200,74 & $(9,05$ & 39,97 & 10,11 & 72,41 & 5,59 \\
\hline AP & 12294,30 & 13,74 & 45,00 & 9,17 & 65,68 & 10,29 \\
\hline PA & 14522,12 & 18,97 & 32,13 & 12,89 & 60,75 & 3,52 \\
\hline RO & 16014,16 & 17,84 & 33,56 & 15,49 & 54,31 & 10,37 \\
\hline RR & 12494,69 & 14,33 & 44,61 & 13,50 & 84,21 & 3,64 \\
\hline TO & 13007,90 & 19,21 & 36,86 & 14,61 & 64,53 & 9,20 \\
\hline Médias & 14727,64 & 15,51 & 38,19 & 12,93 & 66,14 & 7,19 \\
\hline \multicolumn{7}{|c|}{ Nordeste } \\
\hline MA & 9279,17 & 15,36 & 29,24 & 15,87 & 59,63 & 5,40 \\
\hline $\mathrm{PI}$ & 7499,36 & 26,09 & 28,00 & 15,40 & 66,88 & 11,79 \\
\hline CE & 10777,23 & 17,24 & 32,76 & 14,77 & 67,30 & 4,12 \\
\hline $\mathrm{RN}$ & 12637,75 & 13,09 & 34,48 & 13,92 & 77,57 & 5,21 \\
\hline PB & 9148,75 & 15,71 & 30,84 & 18,05 & 74,19 & 5,16 \\
\hline $\mathrm{PE}$ & 12365,95 & 20,82 & 33,72 & 14,54 & 72,02 & 7,72 \\
\hline$A L$ & 10612,16 & 10,64 & 25,62 & 19,40 & 61,98 & 10,36 \\
\hline SE & 14577,61 & 10,74 & 33,33 & 9,85 & 80,65 & 4,90 \\
\hline BA & 12924,04 & 12,40 & 32,88 & 14,43 & 71,98 & 6,51 \\
\hline Médias & 11091,34 & 15,79 & 31,21 & 15,14 & 70,24 & 6,80 \\
\hline \multicolumn{7}{|c|}{ Sudeste } \\
\hline ES & 28549,76 & 18,39 & 40,81 & 9,63 & 83,92 & 6,76 \\
\hline MG & 21570,43 & 17,17 & 37,27 & 11,24 & 84,97 & 1,58 \\
\hline $\mathrm{RJ}$ & 32891,58 & 14,34 & 47,28 & 8,80 & 92,23 & 1,10 \\
\hline SP & 38016,42 & 11,78 & 49,29 & 9,24 & 96,58 & 0,69 \\
\hline Médias & 30257,05 & 15,42 & 43,66 & 9,73 & 89,43 & 2,53 \\
\hline \multicolumn{7}{|l|}{ Sul } \\
\hline PR & 27662,14 & 13,67 & 42,41 & 10,25 & 85,01 & 3,41 \\
\hline SC & 29844,49 & 13,63 & 42,70 & 11,17 & 86,13 & 2,80 \\
\hline RS & 26728,43 & 14,41 & 38,29 & 9,82 & 86,88 & 2,19 \\
\hline Médias & 28078,35 & 13,90 & 41,13 & 10,41 & 86,01 & 2,80 \\
\hline \multicolumn{7}{|c|}{ Centro Oeste } \\
\hline GO & 22347,06 & 16,21 & 40,08 & 12,99 & 74,68 & 7,45 \\
\hline MS & 23172,61 & 18,62 & 38,05 & 12,80 & 69,75 & 7,58 \\
\hline MT & 26386,25 & 23,18 & 39,05 & 15,31 & 65,18 & 8,40 \\
\hline DF & 41869,33 & 10,20 & 56,63 & 7,71 & 95,62 & 2,36 \\
\hline Médias & 28443,81 & 17,05 & 43,45 & 12,20 & 76,31 & 6,45 \\
\hline
\end{tabular}

Fontes: Valores estimados a partir do IBGE dos anos de referência. 
ISAN médio (66,14\%). Roraima (ISAN=54,31\%); Maranhão (ISAN = 59,63\%); e Pará (ISAN $=60,75 \%$ ), são os Estados brasileiros que apresentaram os piores indicadores de saneamento (tal como definidos neste estudo), no período analisado. Por outro lado, São Paulo (ISAN = 96,58\%); Distrito Federal (95,62\%); e Rio de Janeiro (ISAN = $92,23 \%)$ foram aqueles que apresentaram os melhores desempenhos neste indicador. (Tabela 2).

Das evidencias mostradas na Tabela 2 se depreende que os Estados e regiões com maiores produtividades são aqueles que também mostraram os melhores desempenhos nos indicadores de capital humano utilizados neste estudo. Contrariamente, os Estados e regiões com menores desempenhos nos indicadores de capital humano (sobretudo das regiões Nordeste e Norte), são os que apresentaram as menores médias de produtividade, confirmando até aqui as expectativas que nortearam a construção da pesquisa.

\section{RESULTADOS ALCANÇADOS PARA ATINGIR O TERCEIRO OBJETIVO}

Na Tabela 3, construída a partir das evidencias apresentadas na Tabela 2, estão mostradas as freqüências de ocorrências dos CV classificados como "baixos", "médios" e "altos" para as produtividades, escolaridades e ISAN dos Estados. Observa-se que não foi observada a presença de CV "muito alto" de acordo com a hierarquia proposta por Gomes (1985). Foram três (3) os CV estimados e classificados como "altos". Todos foram observados para as produtividades, e foram estimados apara os Estados do Piauí (26,09\%); Mato Grosso (23,18\%) e Pernambuco (20,82\%). Tabela 2 e Tabela 3.

Os coeficientes de variação classificados como "médios" foram observados para as produtividades de 22 Estados e para o DF. Também foram estimados CV de escolaridade classificados como "médios" para 20 Estados. Para o ISAN as heterogeneidades aferidas com "baixas" aconteceram em 22 Estados e no DF. ISAN com CV classificado como "médios" aconteceram em apenas 4 Estados. (Tabela 3).

Assim, se depreende que houve predominância de CV médios entre os indicadores estudados na pesquisa. E que as produtividades e as escolaridades apresentaram as maiores freqüências de CV classificados assim. Pode-se então inferir que no período

Tabela 3: Qualificação dos CV dos Indicadores de acordo com as magnitudes.

\begin{tabular}{l|c|c|c}
\hline \multicolumn{4}{l}{ Classificação de acordo com as magnitudes do CV: Número de Estados } \\
\hline Indicador & CV Baixo & CV Médio & CV Alto \\
\hline Produtividade & 1 & 23 & 3 \\
\hline Escolaridade & 7 & 20 & 0 \\
\hline ISAN & 23 & 4 & 0 \\
\hline Total & 31 & 50 & 3 \\
\hline
\end{tabular}

Fontes: Quadro 1 e Tabela 2. 
avaliado prevaleceram heterogeneidades "médias", na produtividade e na escolaridade dos Estados brasileiros (Tabela 3).

No que se refere aos ISAN estimados para os Estados, observa-se que a sua evolução se deu de forma mais estável, ou com heterogeneidades baixas, haja vista que prevaleceu a categoria de CV "baixo". Como a instabilidade foi aferida no tempo, depreende-se que avanços e recuos em saúde preventiva se deram de forma mais previsível ao longo do tempo, ou com baixa oscilação em torno da média do período avaliado. (Tabela 3).

Das evidencias mostradas na Tabela 2 ainda se depreende que o Distrito Federal apresentou a maior média de produtividade e teve o segundo menor CV (10,20\%) deste indicador. O menor CV para as produtividades foi observado para o Amazonas (9,05\%). Em contraste, o Piauí teve a menor produtividade, e teve também a maior heterogeneidade $(26,09 \%)$ neste indicador no período avaliado (Tabela 2 ).

No geral, aos indicadores de maiores produtividades estão associados menores instabilidades temporais tanto neste indicador como nos indicadores de capital humano. Contrariamente, os Estados com menores produtividades, que tiveram também menores indicadores de capital humano também tiveram esses indicadores evoluindo de forma mais instável no período avaliado.

\section{IMPACTOS DAS DIFERENÇAS DE CAPITAL HUMANO SOBRE DIFERENÇAS DE PRODUTIVIDADE: QUARTO OBJETIVO}

Foram contabilizadas 218 combinações que ensejaram os cálculos das diferenças entre produtividades do trabalho, com os respectivos indicadores do capital humano (escolaridade e ISAN). Nessas combinações as diferenças de produtividades, por construção, são todas positivas. Observando-se o que aconteceu nas diferenças entre escolaridades e ISAN correspondestes, constatou-s que: dos 218 pares de diferenças de escolaridade, em 27 (12,4\%) os valores foram negativos. Por outro lado, dos 218 pares de diferenças do ISAN, em 35 (16,1\%) se observaram valores negativos. Constatou-se também que em apenas 9 pares $(4,1 \%)$ as diferenças associadas à escolaridade e ao ISAN foram simultaneamente negativas. Portanto, prevaleceram diferenças positivas nos indicadores de capital humano afetando as diferenças positivas de produtividades contabilizadas na pesquisa, como esperado.

Feitas essas considerações apresentam-se os resultados encontrados com as estimações dos parâmetros que mostram a relação entre as diferenças de produtividade do trabalho (sempre positivas, por construção) e as diferenças dos indicadores de capital humano. Esses resultados estão apresentados na Tabela 4.

Os resultados mostrados na Tabela 4 confirmam as suposições feitas na construção deste trabalho. Aquelas que sugerem que os diferenciais de produtividade, que aferem as diferenças coletadas pela média deste indicador entre os Estados brasileiros, são afetadas positivamente pelos diferenciais de escolaridade e de indicadores que aferem a saúde preventiva dos estados. 
Tabela 4: Impactos das diferenças das médias dos indicadores de capital humano sobre a as diferenças nas médias de produtividade no DF e nos Estados brasileiros entre 2004 e 2015.

\begin{tabular}{lc|c|c}
\hline Variáveis & Coeficiente & Estatística t & Significância \\
\hline Constante & 4985,549 & 7,819 & 0,000 \\
Diferença de escolaridade & 599,871 & 9,521 & 0,000 \\
Diferença de ISAN & 190,155 & 4,900 & 0,000 \\
R2 ajustado & 0,522 & & \\
\hline
\end{tabular}

Fontes: Valores estimados a partir dos dados do IBGE.

Das evidencias apresentadas na Tabela 4 também se depreende que os impactos das diferenças de escolaridade são maiores do que as estimadas para o ISAN sobre diferenciais de produtividade. Com efeito, a diferença de uma unidade em escolaridade provoca um impacto de $\mathrm{R} \$ 600,00$ no diferencial de produtividade. Por outro lado, variações de uma unidade de diferença no ISAN impactam de R\$194,15 aquelas observadas nas produtividades. (Tabela 4).

\section{CONCLUSŌES}

A conclusão geral confirma que os estados do Nordeste apresentam as menores produtividades entre as cinco regiões brasileiras. Está também no Nordeste o maior percentual da população maior de 15 anos que completou apenas o nível fundamental. Os Estados da região Norte se posicionam em segundo lugar em relação à produtividade. Em relação ao ISAN as maiores carências, em termos médios, estão na região Norte. O Nordeste ocupa a segunda pior posição, com valores não muitos distantes daqueles estimados para o Norte.

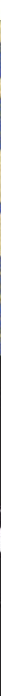


Em contraste, os Estados da região Sudeste mostraram ter as maiores médias de produtividade e os maiores percentuais das populações maiores de 15 anos que estudaram dez anos ou mais. Estão também nos Estados do Sudeste as maiores médias das populações com acesso aos ativos que aferem a saúde preventiva que foram utilizadas no estudo e que foram sintetizados no ISAN.

A pesquisa também partiu da suposição de que as heterogeneidades associadas aos indicadores de capital humano se transmitem e recebem a interferência das heterogeneidades oriundas das produtividades. Essas suposição foi confirmada no estudo e ainda se observou que a heterogeneidade captada na pesquisa paras as produtividades ocorreram em maior quantidade do que as capturadas para o capital humano. Desta evidencia se conclui que devem existir outras variáveis que influenciam na heterogeneidade temporal da produtividade do trabalho, além do capital humano, da forma em que foi aferido neste estudo.

Conclui-se que instabilidades ou heterogeneidades que ocorram no capital humano repercutem sobre a produtividade, tendo em vista que os estados com menores produtividades, no geral, apresentaram maiores instabilidades ao passo que os estados com maiores produtividades o fizeram com menores instabilidades nos indicadores de capital humano.

As diferenças positivas de produtividades foram, em maioria bastante significativa, seguidas de diferenças também positivas nos indicadores de capital humano utilizados na pesquisa. Como se trata de relação causa-efeito, conclui-se que as diferenças de capital humano induzem positivamente as diferenças de produtividade no Distrito Federal e nos Estados brasileiros.

Este estudo também teve entre os seus objetivos mostrar diferenciais entre as produtividades e os indicadores de capital humano no Distrito Federal e nos 26 Estados brasileiros entre os anos de 2004 e 2015. Os resultados obtidos comprovam as suposições que mobilizaram a realização do estado. De fato, diferenciais de capital humano, aferidos neste estudo pela escolaridade e saúde preventiva, afetaram diretamente a produtividade no Distrito Federal e nos Estados brasileiros entre 2004 e 2015.

Resultados promissores que podem ser de utilidade para fazer planejamento que promova desenvolvimento de longo prazo dos Estados brasileiros reduzindo-Ihes as desigualdades. Isso apenas será possível pela elevação da escolaridade e saúde em todos os eles, mas em ritmos diferenciados de aceleração. Aqueles situados nas regiões Norte e Nordeste precisam expandir esses indicadores de forma mais acelerada.

REFERÊNCIAS

AGHION, P; HOWITT, P. A Model of Growth Through Creative Destruction. Econometrica, Washington, v. 60, p. 323-351, 1992.

ALLISON, P. D. 1978. Measures of Inequality. American Sociological Review, v. 43, pp. 865-880. 
ANDERSEN, T.M. Human Capital, Inequality and Growth. Luxemburg. Luxembourg: Publications Office of the European Union. 2015. Disponivel em: https://bit.ly/2vFvntv. Consulta em: 10 Ago. 2019.

BECKER, G. S. Human capital: a theoretical and empirical analysis, with special reference to education. Chicago: The University of Chicago Press, 1964.

BEZERRA, F. N. R. Sustentabilidade da Matriz Energética Brasileira. Dissertação de Mestrado) Universidade Federal do Ceará, Centro de Ciências Agrárias, Programa de Pós-Graduação em Economia Rural, Fortaleza, 2016.

DILLON, W. ; GOLDSTEIN, M. Multivariate analysis: methods and applications. New York: Mc Graw Hill, 1984.

FAO - FOOD AND AGRICULTURE ORGANIZATION OF THE UNITED NATIONS. Policy Impacts on Inequality Simple Inequality Measures, 2006. Disponivel em: https://bit.ly/2Hr8EDK. Acesso em: 05 Maio 2019.

FAO - FOOD AND AGRICULTURE ORGANIZATION OF THE UNITED NATIONS. Refinements to the FAO methodology tor estimation the prevalence of undernourishment indicator. Working Paper N¹4 - 05, 2014. Disponível em: https://bit.ly/38uhJrs. Acesso em: 7 maio 2019.

FÁVERO, L. P.; BELFIORE, P.; SILVA, F. L.; CHN, B. L. Análise de dados: modelagem multivariada para tomada de decisões. Rio de Janeiro. Elsevier Editora Ltda, 2009.

FELEMA, J; RAIHER, A. P; FERRREIRA, R.C. Agropecuária: Desempenho e determinantes da produtividade. Revista de Economia e Sociologia Rrural. Piracicaba- SP. vol. 3, 2013.

FRANÇA, G. N; GASPARINI, C. E; LOUREIRO, P. R. A. Relação entre Escolaridade e Renda na Década de 1990. Encontro Regional de Economia, 2005, Fortaleza. Anais do X Encontro Regional de Economia. Fortaleza: Banco do Nordeste do Brasil, 2005.

GALOR, O. Inequality, Human Capital Formation and the Process of Development. Bonn, Germn. IZA. Discussion Paper n. 6328 .2012. Disponível em: http://ftp.iza.org/dp6328.pdf. Acesso em: 10 ago. 2019.

GARCIA, C.H. Tabelas para classificação do coeficiente de variação. Piracicaba: IPEF, 1989. 12p. (Circular técnica, 171).

GOMES, F.P. Curso de estatística experimental. São Paulo: Nobel, 1985.

GRAY, E.; JACKSON, T.; ZHAO, S. Agricultural productivity: concepts, measurement and factors driving it - a perspective from the ABARES productivity analyses. Rural Industries Research and Development Corporation, Australian Government, n. 10, p. 56, Mar. 2011.

GROSSMAN, G. M; HELPMAN, E. Inovation and grouth in the global Economy. The MIT press, Cambridiege 1991.

HAIR Jr., J. F., ANDERSON, R. E., TATHAM, R. L., BLACK, W. C. Multivariate Data Analysis. Upper Saddle River, NJ: Prentice Hall. (1998).

JOHANSEN, A.L. The Effect of Human Capital on Income Inequality: An Econometric Analysis. Copenhagen. Copenhagen Business School. 2014. Disponivel em: https://bit.ly/2tZJ59Z. Consulta em 10 ago. 2019.

LARIONOVA N.I., VARLAMOVA J.A. Analysis of Human Capital Level and Inequality Interrelation. Mediterranean Journal of Social Sciences. Rome, Italy. Vol 6 No 1. P. 252-255.

LEE, J.W.; LEE, H. Human capital and income inequality. Kasumigaseki, Chiyoda-ku Tokyo 1006008, Japan. Asian Development Bank Institute. 2018. Disponível em: https://bit.ly/2UUBtAu. Consulta em 10 ago. 2019. 
LIRA, J.S. Resiliência da agricultura familiar no Nordeste brasileiro. Fortaleza, CE. Mestrado Acadêmico em Economia Rural (MAER), Universidade Federal do Ceará. Dissertação de Mestrado em Economia Rural. 2016.

LUCAS, R. On the mechanics economic development. Journal of Monetary Economics, v. 12, p. 3-42, 1988.

MENEZES FILHO, N. A; CAMPOS, G.S; KOMATSU, B. K. A evolução da produtividade do trabalho no Brasil. Centro de Políticos públicas INSPER. Policypaper, n 12. Agosto, 2014.

MINCER, J. Investment in Human Capital and Personal Income Distribution. Journal of Political Economy, 1958.

MOREIRA, D. A. Medidas da produtividade na empresa moderna. São Paulo: Pioneira, 1991.

O’REILLY, C. A.; CALDWELL, D. F.; BARNETT W. P. “Work Group Demography, Social Integration, and Turnover." Administrative Science Quarterly. 34:21-37. 1989.

PUNT, C. Measures of Poverty and Inequality: A Reference Paper. PROVIDE Technical Paper 2003:4. Disponivel em: https://bit.ly/2UUA78W. Acesso em: 5 maio 2019.

SCHULTZ, T. W. Reflections on Investment in Man. Journal of Political Economy, v. 70, n. 5, p. 1-8, 1962.

SORENSEN, A.T. Equilibrium Price Dispersion in Retail Markets for Prescription Drugs. Journal of Political Economy, 2000, vol. 108, n. 4 p. $833-850$.

UNITED NATIONS - UN. Human Development Report. New York. 2006.

UNITED NATIONS - UN. Human Development Report. New York. 2014.

VARIAN, H.R. Microeconomia: Uma abordagem moderna. São Paulo (SP). Elsevier. 2015.

VAZ, M. A. B.; PACHECO, P.S; SEIDEL, H.J.; ANSUJ, A.P. Classification of the coefficient of variation to variables in beef cattle experiments. Ciência Rural, Santa Maria, v.47, n. 11, 2017. Disponivel em: https://bit.ly/31Sxp5p. Acesso em: 05 maio 2019.

VIEIRA FILHO, J. E. R. e SILVEIRA, J. M. F. J. Mudança tecnológica na agricultura: uma revisão crítica da literatura e o papel das economias de aprendizado. Revista de Economia e Sociologia Rural, Piracicaba, v. 50, n. 4, p. 721-742, out./dez. 2012.

WIERSEMA, M.; BANTEL, K. "Top Management Team Turnover as an Adaptation Mechanism: The Role of the Environment." Strategic Management Journal. 14, pp. 485-504. 1993.

WOOLDRIDGE, J. M. Econometric Analysis of Cross Section and Panel Data. The MIT Press, Cambridge, MA, 2012.

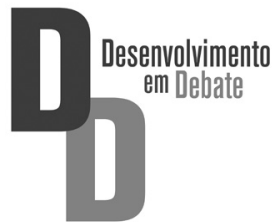

